\title{
Efficacy of tailored second-line therapy of Helicobacter pylori eradication in patients with clarithromycin-based treatment failure: a multicenter prospective study
}

Siya Kong ${ }^{1,2 \dagger}$, Keting Huang ${ }^{1,2 \dagger}$, Jun Wang ${ }^{3 \dagger}$, Xiaoyong Wang ${ }^{4}$, Ningmin Yang ${ }^{5}$, Yu Dong ${ }^{1}$, Ya Zhuang ${ }^{2}$, Yini Dang ${ }^{1,2}$, Guoxin Zhang ${ }^{1,2^{*}+}$ and Feng Ye $\mathrm{e}^{1,2^{*}+}$ (D)

\begin{abstract}
Background: After the failure of clarithromycin- and bismuth-based quadruple therapy (CBQT), levofloxacin- and bismuth-based quadruple therapy (LBQT) is recommended for Helicobacter pylori eradication. We compared the efficacies of second-line tailored bismuth-based quadruple therapy (TBQT) and empirical LBQT.
\end{abstract}

Methods: Patients with CBQT failure were randomly assigned to receive TBQT or LBQT for 14 days. All patients underwent endoscopy for culture-based antibiotic susceptibility testing. Patients in the TBQT group exhibiting levofloxacin susceptibility were randomized to receive amoxicillin, levofloxacin, esomeprazole, and colloidal bismuth pectin (ALEB) or amoxicillin, furazolidone, esomeprazole, and colloidal bismuth pectin (AFEB) for 14 days; patients with levofloxacin resistance received $A F E B$.

Results: From May 2016 to June 2019, 364 subjects were enrolled. Eradication rates were significantly higher in the TBQT group $(n=182)$ than in the LBQT group $(n=182)$ according to both intention-to-treat (ITT) analysis (89.6\% vs. $64.8 \%, \mathrm{P}<0.001)$ and per protocol (PP) analysis (91.1\% vs. 67.8\%, P<0.001). Among patients in the TBQT group with levofloxacin susceptibility, eradication rates were similar in the ALEB $(n=51)$ and AFEB $(n=50)$ subgroups according to both the ITT (86.3\% vs. 90.0\%, P = 0.56) and PP (88.0\% vs. 90.0\%, P = 0.75) analyses. Isolated clarithromycin and levofloxacin resistance rates were $57.7 \%$ and $44.5 \%$, respectively. The total clarithromycin and levofloxacin resistance rate in strains with dual or triple resistance was $35.7 \%$.

Conclusions: TBQT was more effective than $\angle B Q T$ as a second-line strategy after CBQT failure. In the absence of antibiotic susceptibility testing, AFEB therapy might be used as a rescue therapy to eradicate H. pylori and avoid levofloxacin resistance.

Trial registration: Chinese Clinical Trial Registry (www.chictr.org.cn): ChiCTR1900027743.

Keywords: Tailored therapy, Clarithromycin resistance, Second-line therapy, Levofloxacin, Helicobacter pylori

*Correspondence: guoxinz@njmu.edu.cn; fengye@njmu.edu.cn †Siya Kong, Keting Huang and Jun Wang: Joint first authors ${ }^{\dagger}$ Feng Ye and Guoxin Zhang: Joint senior authors

1 Department of Gastroenterology, First Affiliated Hospital of Nanjing Medical University, No. 300 Guangzhou Road, 210029 Nanjing, China

Full list of author information is available at the end of the article

\section{Background}

Helicobacter pylori (H. pylori) infection, a bacterial infection of the stomach, affects approximately $50 \%$ of the global population [1]. H. pylori infection is associated with risks of peptic ulcer, chronic gastritis, intestinal metaplasia, and gastric cancer [2]. Therefore, consensus

(c) The Author(s) 2020. This article is licensed under a Creative Commons Attribution 4.0 International License, which permits use, sharing, adaptation, distribution and reproduction in any medium or format, as long as you give appropriate credit to the original author(s) and the source, provide a link to the Creative Commons licence, and indicate if changes were made. The images or other third party material in this article are included in the article's Creative Commons licence, unless indicated otherwise in a credit line to the material. If material is not included in the article's Creative Commons licence and your intended use is not permitted by statutory regulation or exceeds the permitted use, you will need to obtain permission directly from the copyright holder. To view a copy of this licence, visit http://creativeco mmons.org/licenses/by/4.0/. The Creative Commons Public Domain Dedication waiver (http://creativecommons.org/publicdomain/ zero/1.0/) applies to the data made available in this article, unless otherwise stated in a credit line to the data. 
reports have proposed that $H$. pylori eradication treatment is necessary to prevent these diseases $[2,3]$, particularly among patients who have a family history of gastric cancer in first-degree relatives [4]. Unfortunately, the eradication rates of standard therapy have decreased to alarmingly low levels due to increasing levels of antibiotic resistance; eradication is particularly difficult when the clarithromycin resistance rate is greater than $15 \%$ in the local region $[5,6]$.

In recent decades, the resistance rates to clarithromycin, levofloxacin, and metronidazole have reached $22 \%$, $19 \%$ and $78 \%$, respectively, in China [7]. The prevalence of resistance to clarithromycin (22.9-37\%), levofloxacin (5.7-34.6\%), and metronidazole (14.4-93.2\%) has increased significantly over time and varies substantially among countries in the Asia-Pacific region $[8,9]$. The success rate of standard $H$. pylori eradication treatment has decreased to $60 \%$ due to increasing levels of unrecognized antibiotic resistance, high intragastric bacterial loads before treatment, poor compliance, and the rapid metabolism of proton pump inhibitors (PPIs) [5, 10]. The use of antibiotic susceptibility-guided therapy has therefore been proposed as a countermeasure for resistance-associated treatment failure and the emergence of antibiotic resistance [11]. In regions with a high clarithromycin resistance rate $(26.12 \%)$, the administration of tailored colloidal bismuth pectin-containing quadruple therapy to patients for 14 days has achieved eradication rates of $85.99 \%$ and $91.22 \%$ according to intention-totreat (ITT) and per protocol (PP) analyses, respectively [12]. The eradication rates of susceptibility-guided therapies have been reported to exceed $90 \%$, according to PP analyses, in regions with high clarithromycin resistance rates $(>15 \%)$, even when these therapies were used as rescue treatments [2, 11-14].

After the failure of clarithromycin-based triple therapy, colloidal bismuth pectin-containing quadruple therapy or fluoroquinolone-containing triple or quadruple therapy is recommended as a second-line treatment by the Maastricht V/Florence Consensus Report [15]. However, patients with treatment failure are more likely to be infected with metronidazole-, levofloxacin-, and/or clarithromycin-resistant bacteria [16, 17]; thus, the efficacy of levofloxacin has decreased in recent years. Hence, an improved rescue therapy regimen for $H$. pylori infection following the failure of first-line clarithromycincontaining eradication treatment is needed. Based on data from pilot studies, the administration of tailored treatment as a second-line rescue treatment for $H$. pylori infection achieved a very high eradication rate (95\%) [11]. However, the benefits of tailored second-line treatment remain unclear $[15,18]$. Currently, researchers have not yet determined whether the tailored quadruple regimen is superior to standard levofloxacin-containing quadruple therapy, and its efficacy as a rescue treatment for patients with failure of clarithromycin-based quadruple therapy remains to be determined. Therefore, in this prospective, randomized clinical trial, we evaluated the possibility of using levofloxacin- or furazolidone-based quadruple therapy as a universal rescue treatment. In addition, we investigated the efficacy of tailored bismuth-based quadruple therapy (TBQT; containing levofloxacin or furazolidone) and compared it with empirical levofloxacin- and bismuth-based quadruple therapy (LBQT) for 14 days as a second-line treatment.

\section{Methods \\ Participants}

This study was designed as a multicenter, open-label, randomized, controlled trial and was conducted between May 2016 and June 2019 in the clinics of the First Affiliated Hospital of Nanjing Medical University, Changzhou Second People's Hospital Affiliated with Nanjing Medical University, and Jinhu County People's Hospital. Consecutive patients with a failure of clarithromycin-based eradication treatment for $H$. pylori within the previous 6 months were enrolled. Patients were eligible if they had a confirmed $H$. pylori infection based on the ${ }^{13} \mathrm{C}$-urea breath test (UBT). The following exclusion criteria were used: (1) patients who had received $H$. pylori eradication treatment more than once; (2) patients who had been treated with antibiotics, colloidal bismuth pectin, $\mathrm{H}_{2}$ receptor inhibitors, or PPIs within the previous 4 weeks; (3) patients with a history of fluoroquinolone drug treatment, particularly levofloxacin; (4) patients with serious diseases, such as severe cardiopulmonary and liver dysfunction; and (5) patients with an allergy to the study drugs. Each patient provided written informed consent, and the study protocol was approved by the ethics committee of each center. The trial was registered at the Chinese Clinical Trial Registry (ChiCTR) with a registration number of ChiCTR1900027743.

\section{Study groups, trial design, and procedures}

According to the consensus on the eradication of H. pylori in China [10], we used the following regimens: TBQT consisted of amoxicillin (1000 mg twice daily) + levofloxacin (500 $\mathrm{mg}$ once daily) or furazolidone (100 mg twice daily)+esomeprazole $(20 \mathrm{mg}$ twice daily) + colloidal bismuth pectin (220 mg twice daily). LBQT consisted of amoxicillin (1000 mg twice daily) + levofloxacin (500 mg once daily) + esomeprazole (20 mg twice daily) + colloidal bismuth pectin $(220 \mathrm{mg}$ twice daily). ALEB also consisted of amoxicillin (1000 mg twice daily) + levofloxacin (500 mg once daily) + esomeprazole $(20 \mathrm{mg}$ twice daily) + colloidal bismuth pectin 
(220 mg twice daily). The difference between LBQT and ALEB therapy is that ALEB therapy was based on the results of antibiotic susceptibility testing. LBQT therapy was administered to all patients in the LBQT group, regardless of the results of antibiotic susceptibility testing, and the doctors administering this treatment did not know the results of the susceptibility test. AFEB therapy consisted of amoxicillin (1000 mg twice daily) + furazolidone (100 mg twice daily) + esomeprazole (20 mg twice daily) + colloidal bismuth pectin (220 mg twice daily). All treatment regimens were administered for 14 days.

Before enrollment, patients underwent ${ }^{13} \mathrm{C}$-UBTs for the detection of $H$. pylori infection. Eligible subjects underwent endoscopy for antibiotic susceptibility testing. They were then assigned in a 1:1 ratio to the TBQT group or the LBQT group using a randomized digital table. In the TBQT group, antibiotic selection was based on the results of the susceptibility tests as follows: patients with levofloxacin-sensitive strains were further randomly assigned in a 1:1 ratio to either the ALEB therapy subgroup or the AFEB therapy subgroup. Patients with levofloxacin-resistant strains were assigned to the AFEB therapy subgroup. Successful eradication was evaluated using a ${ }^{13} \mathrm{C}$-UBT performed at least 4 weeks after the treatment ended. Patients in the LBQT group for whom treatment failed in our study were able to undergo their next round of treatment according to the results of the antibiotic susceptibility test performed at the start of this study. Positive results ( $\geq 4$ units) were defined as $H$. pylori treatment failure. Patient compliance and adverse events were assessed through interviews.

H. pylori isolation, culture, and antibiotic susceptibility test $H$. pylori isolates were obtained from biopsy specimens harvested from the lesser gastric antrum and the greater gastric curvature during endoscopy. Isolation of $H$. pylori and antibiotic susceptibility testing were conducted at the Hangzhou Zhiyuan Medical Inspection Institute. The minimum inhibitory concentrations were defined as follows: amoxicillin $\geq 2 \mu \mathrm{g} / \mathrm{mL}$, clarithromycin $\geq 1 \mu \mathrm{g} / \mathrm{mL}$, levofloxacin $\geq 2 \mu \mathrm{g} / \mathrm{mL}$, furazolidone $\geq 2 \mu \mathrm{g} / \mathrm{mL}$, and metronidazole $\geq 8 \mu \mathrm{g} / \mathrm{mL}[19,20]$. A standard $H$. pylori strain (ATCC43504) was used for quality control.

\section{CYP2C19 genetic polymorphism}

We used the PCR-restriction fragment length polymorphism method to detect the genotypes of variant CYP2C19 alleles ("2, *3, and "17) [21]. DNA was extracted from the gastric mucosal samples by using a QIAamp mini kit (Qiagen, Düsseldorf, Germany). Patients were divided into four groups according to the genotype identified by testing for the CYP2C19 wild-type (CYP2C19 *1) gene and the three mutated alleles (CYP2C19*2,
CYP2C19 *3, and CYP2C19 *17). Patients without a mutation $\left(* 1 /{ }^{*} 1\right)$ were defined as the homozygous extensive metabolizer (EM) group, patients with one mutation $(* 1 / 22$ or $* 1 / * 3)$ were defined as the heterozygous intermediate metabolizer (IM) group, patients with two mutations $\left(* 2 /{ }^{*} 2,{ }^{*} 3 / * 3\right.$, or $\left." 2 /{ }^{*} 3\right)$ were defined as the poor metabolizer (PM) group, and patients with the heterozygous CYP2C19 * $1 /{ }^{*} 17$ or homozygous CYP2C19 *17/"17 genotype were designated as the ultra-rapid metabolizer (UM) group [22].

\section{Statistical analysis}

The primary outcomes were the eradication rate and side effect rate in the TBQT group and LBQT group. The secondary outcomes were the eradication rate in the ALEB therapy subgroup, combined AFEB therapy subgroup, and $L B Q T$ group, and the side effect rate in the ALEB therapy subgroup and combined AFEB therapy subgroup. A $P$ value $<0.05$ was considered significant. The eradication rate and patient-reported side effect rate were examined using ITT and PP analyses. Subjects who violated the study protocol, for example, by not taking at least $80 \%$ of the treatment drugs, were excluded from the PP analysis. Categorical variables are reported as percentages and were analyzed using the $\chi^{2}$ test. Continuous variables were compared between groups using the Student $t$ test. A P value $<0.05$ was considered significant. The Statistical Package for the Social Sciences software (version 25.0; SPSS, Inc., Chicago, IL, USA) version 25.0 was used for the statistical analyses.

\section{Results}

\section{Participants}

Six hundred thirty-five patients were screened during the study period. Of these patients, 271 did not meet the selection criteria, including 93 patients with prior fluoroquinolone use and 106 patients with a history of more than one anti- $H$. pylori treatment regimen; the remaining 364 patients were randomly assigned to the TBQT $(n=182)$ or LBQT group $(n=182)$. In the TBQT group, patients without levofloxacin resistance were randomly assigned in a 1:1 ratio to either the ALEB therapy subgroup $(n=51)$ or the AFEB therapy subgroup $(n=50)$. Patients in the TBQT group with levofloxacin resistance were assigned to the AFEB therapy group $(n=81)$. All recruited patients received complete follow-up (Fig. 1).

The baseline characteristics of the participants were well balanced between the groups (Table 1). Eleven of the 364 patients were excluded from the PP analysis; 3 of these patients were lost to follow-up, 6 violated the study protocol because of adverse effects, and 2 patients refused to undergo follow-up ${ }^{13} \mathrm{C}$-UBTs. 


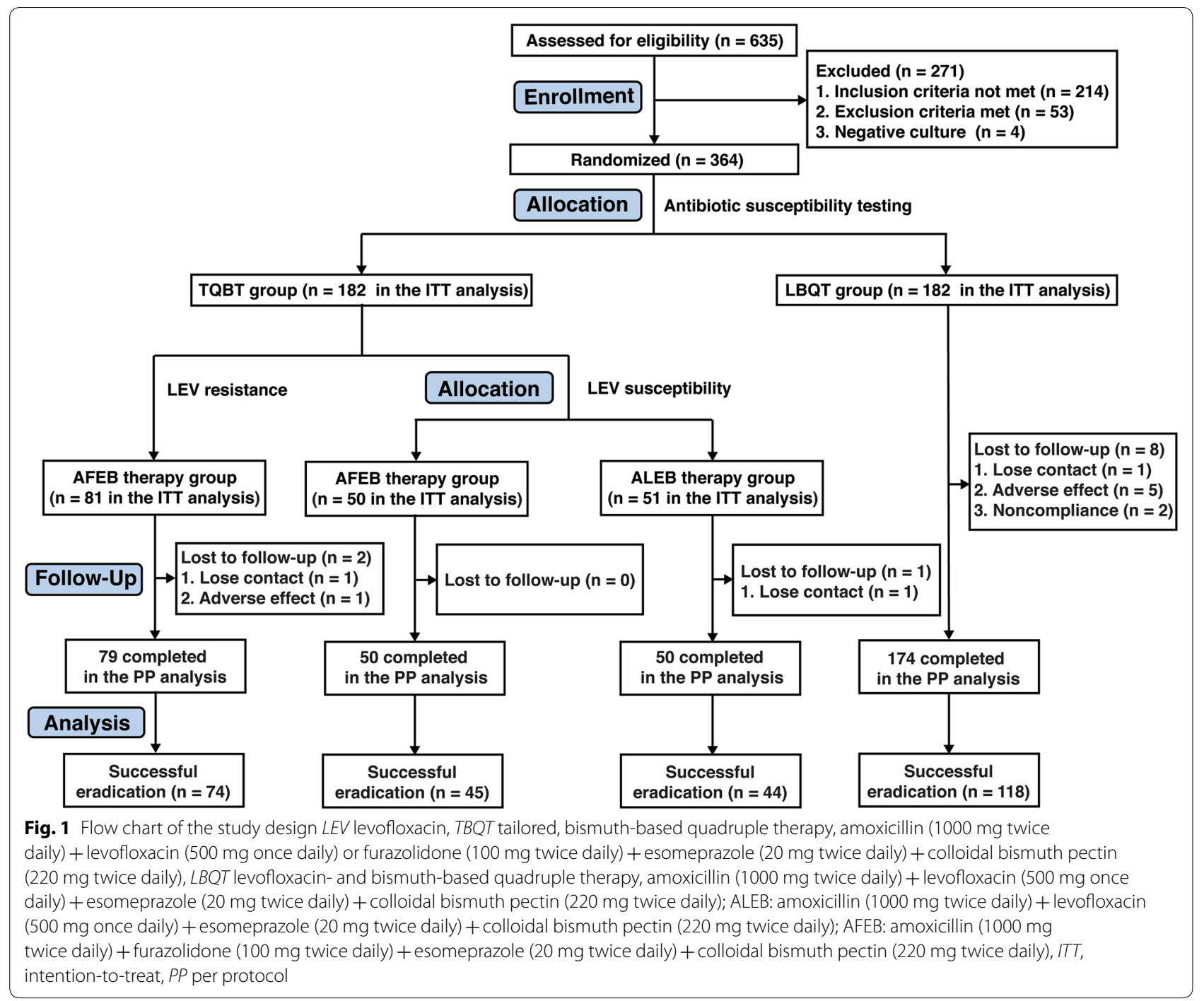

Table 1 Characteristics of the participants

\begin{tabular}{llll}
\hline Characteristic & TBQT-AFEB group $(\mathbf{n = 1 3 2 )}$ & TBQT-ALEB group $(\mathbf{n}=\mathbf{5 0})$ & LBQT group $(\mathbf{n}=\mathbf{1 8 2})$ \\
\hline Age $(y r s)$, mean \pm SD & $47.1 \pm 11.2$ & $47.2 \pm 12.1$ & $48.09 \pm 13.6$ \\
Male & $52 \%(68 / 132)$ & $52 \%(26 / 50)$ & $47 \%(86 / 182)$ \\
Smoker & $19 \%(25 / 132)$ & $20 \%(10 / 50)$ & $12 \%(21 / 182)$ \\
Drinker & $20 \%(26 / 132)$ & $20 \%(10 / 50)$ & $26 \%(48 / 182)$ \\
BMl $\left(\mathrm{kg} / \mathrm{m}^{2}\right)$ & $23.21 \pm 2.96$ & $22.70 \pm 3.20$ & $22.69 \pm 3.54$ \\
Family history & $22 \%(29 / 132)$ & $22 \%(11 / 50)$ & $19 \%(34 / 182)$ \\
Peptic ulcer disease & $20 \%(27 / 132)$ & $20 \%(10 / 50)$ & $15 \%(28 / 182)$ \\
Chronic atrophic gastritis with intestinal meta- & $36 \%(47 / 132)$ & $36 \%(18 / 50)$ & $39 \%(71 / 182)$ \\
$\quad$ plasia & & & \\
Gastrointestinal symptoms & $38 \%(50 / 132)$ & $38 \%(19 / 50)$ & $41 \%(75 / 182)$ \\
Clarithromycin resistance & $57 \%(75 / 132)$ & $56 \%(28 / 50)$ & $59 \%(107 / 182)$ \\
\hline
\end{tabular}

$T B Q T$ tailored, bismuth-based quadruple therapy, amoxicillin + levofloxacin or furazolidone + esomeprazole + colloidal bismuth pectin, $L B Q T$ levofloxacin- and bismuth-based quadruple therapy, amoxicillin + levofloxacin + esomeprazole + colloidal bismuth pectin, BMI body mass index 


\section{Antibiotic resistance}

The metronidazole-, clarithromycin-, and levofloxacinresistance rates were $91.8 \%$ (334/364), 57.7\% (210/364), and $44.5 \%$ (162/364), respectively (Fig. 2a). In our population, multidrug-resistant strains were predominant. Only $3.3 \%$ (12/364) of $H$. pylori isolates were susceptible to all three tested antibiotics; the remaining $96.7 \%$ of $H$. pylori isolates showed single, dual, or triple resistance to clarithromycin, levofloxacin, and metronidazole. Single antibiotic resistance was most commonly observed for metronidazole (91.8\%), while dual antibiotic resistance (32.4\%) was most commonly observed for metronidazole and clarithromycin. The pooled prevalence of clarithromycin, metronidazole, and levofloxacin resistance was 32.4\% (118/364; Fig. 2b). In addition, the total clarithromycin and levofloxacin resistance rate among strains with dual (clarithromycin and levofloxacin, $n=12 / 118$ ) or triple (clarithromycin, levofloxacin and metronidazole, $\mathrm{n}=118 / 118)$ resistance was $35.7 \%$ (130/364).

\section{Clinical efficacy}

The eradication rates in the TBQT and LBQT groups were $89.6 \%$ and $64.8 \%(\mathrm{P}<0.001)$ in the ITT analysis and $91.1 \%$ and $67.8 \%(\mathrm{P}<0.001)$ in the $\mathrm{PP}$ analysis, respectively (Table 2 ). The eradication rate in the TBQT group was significantly higher than in the LBQT group $(\mathrm{P}<0.001)$.

\section{Clinical efficacy in the TBQT group}

The eradication rates in the levofloxacin-susceptible ALEB therapy subgroup and AFEB therapy subgroup
Table $2 H$. pylori eradication rates in the TBQT and LBQT groups

\begin{tabular}{llll}
\hline Eradication rate & $\begin{array}{l}\text { TBQT group } \\
(\mathbf{n = 1 8 2})\end{array}$ & $\begin{array}{l}\text { LBQT group } \\
(\mathbf{n = 1 8 2})\end{array}$ & P value \\
\hline ITT analysis & $163 / 182(89.6 \%)$ & $118 / 182(64.8 \%)$ & $<0.001$ \\
PP analysis & $163 / 179(91.1 \%)$ & $118 / 174(67.8 \%)$ & $<0.001$ \\
\hline
\end{tabular}

$T B Q T$ tailored, bismuth-based quadruple therapy, amoxicillin + levofloxacin or furazolidone + esomeprazole + colloidal bismuth pectin $\angle B Q T$ levofloxacin- and bismuth-based quadruple therapy. amoxicillin + levofloxacin + esomeprazole + colloidal bismuth pectin, ITT intention-to-treat, $P P$ per protocol

were $88.0 \%$ and $90.0 \%(\mathrm{P}=0.75)$, respectively, in the $\mathrm{PP}$ analysis and $86.3 \%$ and $90.0 \%(P=0.56)$, respectively, in the ITT analysis (Fig. 3). There was no significant difference in the eradication rate among the three subgroups in the TBQT group in the ITT $(\mathrm{P}=0.64)$ and PP analyses $(\mathrm{P}=0.52)$. In all, in the levofloxacin-susceptible group, the ALEB and AFEB regimens achieved similar efficacy rates.

\section{Clinical efficacy in the ALEB therapy subgroup, AFEB therapy subgroup, and LBQT group}

The eradication rates in the ALEB therapy subgroup and LBQT group were $86.3 \%$ and $64.8 \%(\mathrm{P}=0.003)$, respectively, in the ITT analysis and $88.0 \%$ and $67.8 \%$ $(\mathrm{P}=0.005)$, respectively, in the PP analysis (Table 3$)$. The eradication rates in the combined AFEB therapy subgroup (i.e., including all patients in the levofloxacin-susceptible and levofloxacin-resistant groups who received
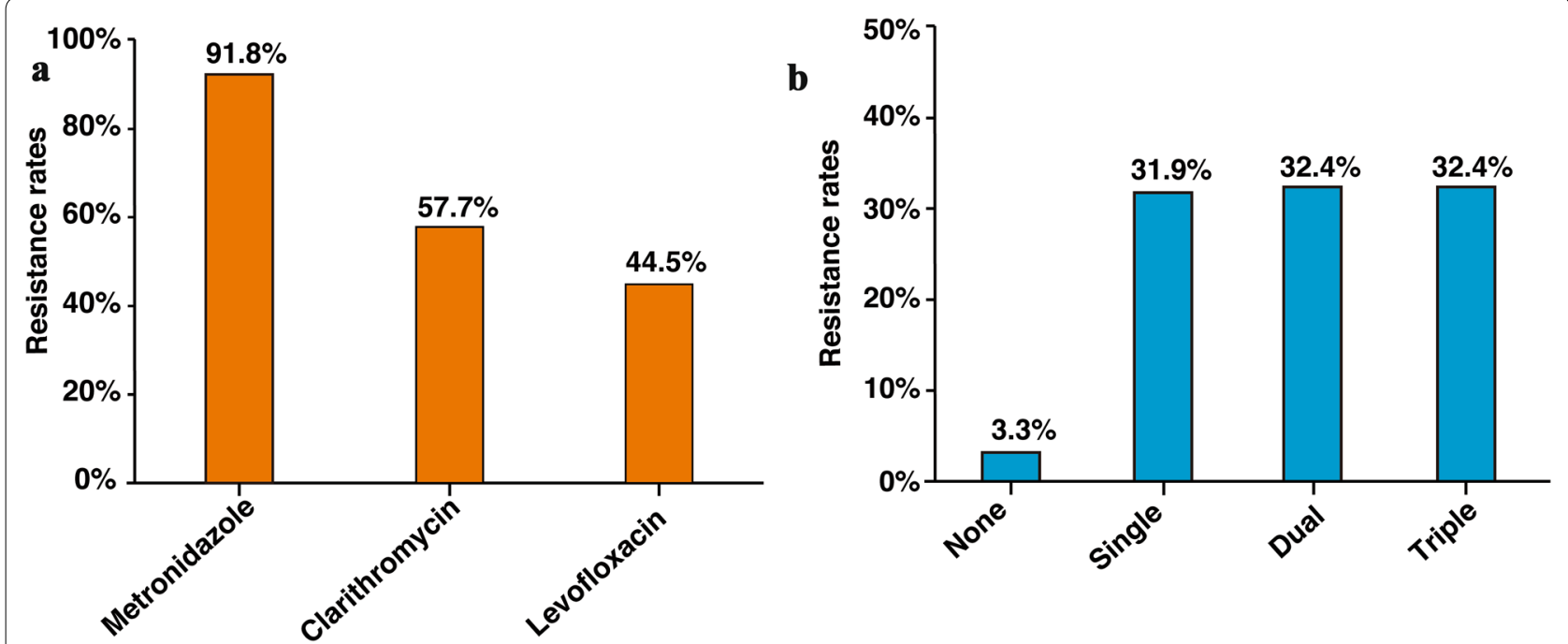

Fig. 2 Antibiotic resistance rates a The metronidazole-, clarithromycin-, and levofloxacin-resistance rates in the total population. $\mathbf{b}$ Multidrug-resistant infections. None, Single, Dual, and Triple indicate no, single, dual, and triple resistance to clarithromycin, levofloxacin, and metronidazole in the TBQT group, respectively. Multidrug-resistant strains were predominant, with $64.8 \%$ of strains showing dual or triple resistance 


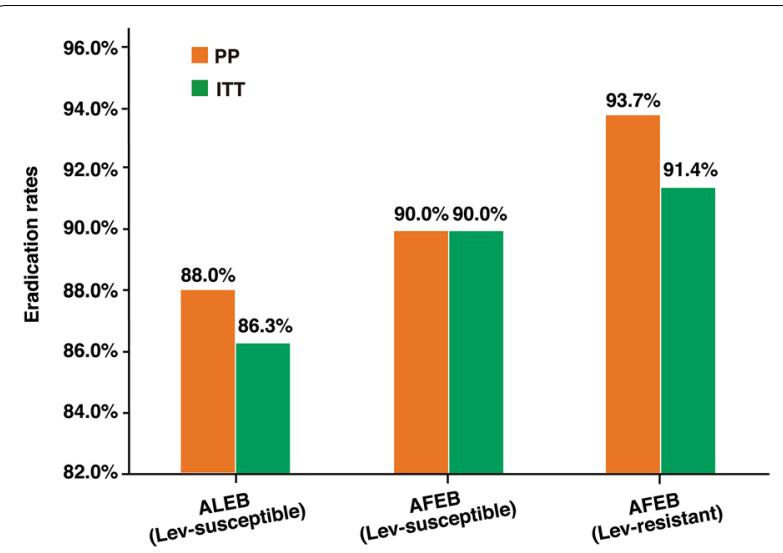

Fig. 3 H. pylori eradication rates in the TBQT group ALEB/AFEB: amoxicillin (1000 mg twice daily) + levofloxacin (500 mg once daily) or furazolidone (100 mg twice daily) + esomeprazole (20 mg twice daily) + colloidal bismuth pectin (220 mg twice daily) Lev: levofloxacin Clinical efficacies in the levofloxacin-susceptible group (ALEB therapy subgroup and AFEB therapy subgroup) and levofloxacin-resistant group (AFEB therapy subgroup) according to the ITT and PP analyses

AFEB therapy) and LBQT group were $90.8 \%$ and $64.8 \%$ $(\mathrm{P}=0.000)$, respectively, in the ITT analysis and $92.2 \%$ and $67.8 \%(\mathrm{P}=0.000)$, respectively, in the $\mathrm{PP}$ analysis. A significant difference in the eradication rate was not observed between the ALEB therapy subgroup and the combined AFEB therapy subgroup in the ITT $(\mathrm{P}=0.366)$ and $\mathrm{PP}$ analyses $(\mathrm{P}=0.371)$.

\section{Adverse events}

The safety analysis included 361 participants. Three patients were lost to follow-up. The incidence of adverse events in the TBQT and LBQT groups was $40.0 \%$ $(72 / 180)$ and $45.9 \%(83 / 181 ; \mathrm{P}=0.26)$, respectively. One participant in the levofloxacin-resistant group who received AFEB therapy and five participants in the LBQT group violated the study protocol due to abdominal discomfort, nausea, and dizziness. The incidence of adverse events did not significantly differ between the groups (Additional file 1: Table S1). In the TBQT group, 132 patients received AFEB therapy (i.e., including all patients in the levofloxacin-susceptible and levofloxacin-resistant groups who received AFEB therapy), and the side effects of AFEB therapy were not more severe than those of LBQT and ALEB therapy (Additional file 1: Table S2).

\section{Analysis stratified according to CYP2C19 polymorphisms}

Of the 364 samples, 28 were insufficient. Thus, 336 samples were subjected to the analysis of CYP2C19 polymorphisms, and $0.9 \%(3 / 336)$ of patients were UMs, $46.4 \%$ (156/336) were EMs, 39.3\% (132/336) were IMs, and $13.4 \%$ (45/336) were PMs (Supplementary Figure S1).

The eradication rates among EMs, IMs, and PMs were 78.8\% (123/156), 81.1\% (107/132), and 84.4\% (38/45), respectively. As shown in Additional file 1: Table S3, no significant difference in the eradication rate was observed among the different CYP2C19 polymorphism-based subgroups $(\mathrm{P}=0.69)$.

\section{Discussion}

Physicians must consider the patient's previous exposure to antibiotics to optimize the management of $H$. pylori treatment. In this prospective, randomized trial, clarithromycin-containing therapy had been administered to the included patients as a first-line treatment. We therefore selected AFEB and ALEB therapy as second-line regimens for $H$. pylori-positive patients. We confirmed that TBQT (AFEB or ALEB) was more effective than LBQT under conditions of a high prevalence of antibiotic resistance, according to both the ITT $(86.3 \%$ vs. $64.8 \%)$ and PP analyses ( $88.0 \%$ vs. $67.8 \%)$. However, one systematic review has suggested that the evidence was too limited to support the generalized use of susceptibility-guided therapy as a rescue treatment for $H$. pylori infection because of unclear antibiotic resistance [18]. In our study, the levofloxacin-resistance rate was $44.5 \%$, consistent with previously reported rates of $34.5-$ $54.8 \%$ in China [23]. In addition, the prevalence of dual clarithromycin and levofloxacin resistance in the whole cohort was $35.7 \%$. Thus, if levofloxacin- or clarithromycin-containing quadruple therapy was to be administered as a second-line therapy to patients who had previously

Table 3 Helicobacter pylori eradication rates in the ALEB therapy subgroup, combined AFEB therapy subgroup, and LBQT group

\begin{tabular}{|c|c|c|c|}
\hline Eradication rate & ALEB therapy subgroup $(n=51)$ & $\begin{array}{l}\text { Combined AFEB therapy subgroup } \\
(n=131)\end{array}$ & LBQT group $(n=182)$ \\
\hline ITT analysis & $44 / 51(86.3 \%)$ & 119/131 (90.8\%) & $118 / 182(64.8 \%)$ \\
\hline PP analysis & $44 / 50(88.0 \%)$ & $119 / 129(92.2 \%)$ & $118 / 174(67.8 \%)$ \\
\hline
\end{tabular}

ALEB (susceptibility-guided therapy) amoxicillin + levofloxacin + esomeprazole + colloidal bismuth pectin, $L B Q T$ levofloxacin- and bismuthbased quadruple therapy not guided by antibiotic susceptibility, amoxicillin + levofloxacin + esomeprazole + colloidal bismuth pectin, $A F E B$ amoxicillin + furazolidone + esomeprazole + colloidal bismuth pectin, ITT intention-to-treat, PP per protocol 
experienced failure of clarithromycin-containing treatment, the possibility of treatment failure would be very high. However, the use of an antibiotic susceptibilityguided approach helped achieve more satisfactory results with second-line levofloxacin-containing treatment, with eradication rates exceeding $85 \%$ in our study. In patients without levofloxacin resistance, the ALEB regimen achieved an efficacy similar to AFEB therapy. In general, most guidelines strongly recommend susceptibility-guided therapy under the circumstances of prior inappropriate antibiotic use and widespread resistance development [15]. The main factor resulting in failure of H. pylori eradication treatment is presumed to be antibiotic resistance [24].

Cure rates greater than $90-95 \%$ should be expected with antimicrobial therapy for $H$. pylori infection [2]. Compared to other antibiotics used as second-line treatments, metronidazole has achieved excellent cure rates $(>90 \%)$ in Japan $[18,25]$. The success rate of tetracyclines or clarithromycin is also greater than $90 \%$ in Taiwan and other regions [26-29]. However, second-line H. pylori treatments that achieve cure rates greater than $90 \%$ are extremely heterogeneous [18]. The administration of metronidazole and clarithromycin-containing therapy as a rescue regimen was expected to be inefficient in our study because of the high antibiotic resistance rate. In our study population, when using the susceptibility-guided approach, only AFEB therapy yielded efficacy rates greater than $90 \%$ in patients with a history of clarithromycin treatment. Levofloxacin, a fluoroquinolone-based antibiotic, has a lower resistance rate than clarithromycin [5]. Bismuth- and levofloxacin-containing quadruple therapy should be reserved as an effective ( $\geq 90 \%$ success rate) second-line strategy for patients who have experienced one treatment failure [30, 31]. However, levofloxacin resistance has increased since the restriction of macrolides [32], and thus it is markedly less effective in fluoroquinolone-containing triple therapies than other agents [33]. In our previous study, we compared the efficacy of levofloxacin-based regimens as first-line anti- $H$. pylori treatments and found a low success rate (78\%) [34]. Hence, AFEB therapy might be the preferred regimen in regions where bacterial susceptibility data are not available to avoid increased levofloxacin resistance [35, 36]. Furazolidone is an effective drug for $H$. pylori eradication; it has a low resistance rate against $H$. pylori and is available in many regions [7]. According to some studies, furazolidone-based quadruple therapy, which has an $88.2 \%$ eradication rate, is an efficacious rescue strategy in patients with a previous eradication therapy failure [35].

In addition, we used esomeprazole in this study, and previous study have reported that CYP2P19 polymorphisms do not influence $H$. pylori eradication rates when esomeprazole or rabeprazole is administered [37]. Consistent with the previous study, we did not observe any significant effect of CYP2C19 polymorphisms on the efficacy of eradication treatment (Additional file 1: Figure S1 and Table S3).

This study has some limitations. First, the relatively small sample size $(n=51)$ in the ALEB therapy group prevented us from clearly explaining the efficacy of levofloxacin. Further studies will be needed to reach an evidence-based conclusion. Second, the patients from the TBQT group were assigned to subgroups according to the results of the antibiotic susceptibility tests; thus, this study was not a completely double-blind study. Fourth, the low BMI $\left(<24 \mathrm{~kg} / \mathrm{m}^{2}\right)$ of our study population may produce a possible selection bias; the next step will be to perform further screening of a patient population with a high BMI.

\section{Conclusions}

In conclusion, second-line levofloxacin-containing quadruple therapy exhibited unacceptable therapeutic efficacy in patients with a failure of clarithromycin-based first-line treatment. The administration of a second-line levofloxacin-containing treatment using a susceptibilityguided approach, however, displayed acceptable efficacy. AFEB therapy was recommended as a rescue regimen in regions where bacterial susceptibility data were not available to avoid increased levofloxacin resistance.

\section{Supplementary information}

Supplementary information accompanies this paper at https://doi. org/10.1186/s13099-020-00378-1.

Additional file 1: Figure S1. CYP2C19 polymorphism rates. Table S1. Adverse events in patients undergoing eradication therapy. Table S2. Adverse events in patients undergoing eradication therapy. Table S3. Analysis according to CYP2C19 polymorphisms.

\section{Abbreviations}

H. pylori: Helicobacter pylori; PPIs: Proton pump inhibitors; ITT: Intention-totreat; PP: Per protocol; CBQT: Clarithromycin- and bismuth-based quadruple therapy; TBQT: Tailored bismuth-based quadruple therapy; LBQT: Levofloxacin- and bismuth-based quadruple therapy, amoxicillin (1000 mg twice daily) + levofloxacin (500 mg once daily) + esomeprazole (20 mg twice daily) + colloidal bismuth pectin (220 mg twice daily); ALEB: Amoxicillin (1000 mg twice daily) + levofloxacin (500 mg once daily) + esomeprazole (20 mg twice daily) + colloidal bismuth pectin (220 mg twice daily); AFEB: Amoxicillin (1000 mg twice daily) + furazolidone (100 mg twice daily) + esomeprazole (20 mg twice daily) + colloidal bismuth pectin ( $220 \mathrm{mg}$ twice daily); UBT: Urea breath test; EM: Extensive metabolizer; IM: Intermediate metabolizer; PM: Poor metabolizer; UM: Ultra-rapid metabolizer.

\section{Acknowledgements}

None.

\section{Authors' contributions}

SK, KH, JW, XW, NY, YD, YZ, YND, GZ and FY all contributed to this study. Study concept and design: GZ and FY. Acquisition of data: SK, KH, JW, XW, NY, YD,

YZ, and YND. Data analysis and interpretation: FY, SK, KH and JW. Statistical 
analysis: FY. Drafting of the manuscript: SK, KH and FY. Critical revision of the manuscript for important intellectual content: GZ and FY. Preparation of the figures and tables: SK. Obtained funding: GZ and FY. Study supervision: GZ and FY. All authors had full access to all of the data and approved the final version of this manuscript. All authors take responsibility for the integrity of the data and the accuracy of the data analysis. All authors read and approved the final manuscript.

\section{Funding}

This study was supported in part by the National Natural Science Foundation of China (Nos. 81500431, 81770561, and 81970499), Natural Science Foundation of Jiangsu Province (No. BK20151039), Six Talent Peaks Project in Jiangsu Province (No. 2018-WSW-003), and Jiangsu Province Leading Talents and Innovation Team (No. CXTDA2017033). The funding agencies did not have a role in the design of the study or the collection, analysis, and interpretation of data.

\section{Availability of data and materials}

The datasets used and/or analyzed during the current study are available from the corresponding author upon reasonable request.

\section{Ethical approval and consent to participate}

The study protocol was reviewed and approved by the ethics committees of the First Affiliated Hospital of Nanjing Medical University, Changzhou Second People's Hospital Affiliated with Nanjing Medical University, and Jinhu County People's Hospital. The trial was registered at the Chinese Clinical Trial Registry (ChiCTR) with a registration number of ChiCTR1900027743. It was registered retrospectively because Feng Ye went abroad for further studies and failed to continue this study.

\section{Consent for publication}

Not applicable.

\section{Competing interests}

The authors have no conflicts of interest to declare.

\begin{abstract}
Author details
1 Department of Gastroenterology, First Affiliated Hospital of Nanjing Medical University, No. 300 Guangzhou Road, 210029 Nanjing, China. ${ }^{2}$ First Clinical Medical College of Nanjing Medical University, Nanjing, China. ${ }^{3}$ Department of Gastroenterology and Hepatology, Jinhu County People's Hospital, Huaian, China. ${ }^{4}$ Department of Gastroenterology, Changzhou Second People's Hospital Affiliated to Nanjing Medical University, Changzhou, China. ${ }^{5}$ Jiangsu Zhiyuan Inspection Medical Institute, Nanjing, China.
\end{abstract}

Received: 11 July 2020 Accepted: 19 August 2020

Published online: 29 August 2020

\section{References}

1. Sjomina O, Pavlova J, Niv Y, Leja M. Epidemiology of Helicobacter pylori infection. Helicobacter. 2018;23(1):e12514.

2. Chey WD, Leontiadis Gl, Howden CW, Moss SF. Correction: ACG clinical guideline: treatment of helicobacter pylori infection. Am J Gastroenterol. 2018;113(7):1102.

3. Du Y, Zhu H, Liu J, Li J, Chang X, Zhou L, Chen M, Lu N, Li Z. Consensus on eradication of Helicobacter pylori and prevention and control of gastric cancer in China (2019, Shanghai). J Gastroenterol Hepatol. 2019;35(4):624-9.

4. Choi IJ, Kim CG, Lee JY, Kim YI, Kook MC, Park B, Joo J. Family History of Gastric Cancer and Helicobacter pylori Treatment. New Eng J Med. 2020;382(5):427-36.

5. Thung I, Aramin H, Vavinskaya V, Gupta S, Park JY, Crowe SE, Valasek MA. Review article: the global emergence of Helicobacter pylori antibiotic resistance. Aliment Pharmacol Ther. 2016;43(4):514-33.

6. Bjorkman DJ, Steenblik M. Best practice recommendations for diagnosis and management of Helicobacter pylori-synthesizing the Guidelines. Current Treatment Options Gastroenterol. 2017;15(4):648-59.

7. Liu DS, Wang YH, Zeng ZR, Zhang ZY, Lu H, Xu JM, Du YQ, Li Y, Wang JB, $\mathrm{Xu}$ SP, et al. Primary antibiotic resistance of Helicobacter pylori in Chinese patients: a multiregion prospective 7-year study. Clin Microbiol Infect. 2018;24(7):780.e785-8.

8. De Francesco V, Giorgio F, Hassan C, Manes G, Vannella L, Panella C, lerardi E, Zullo A. Worldwide H. pylori antibiotic resistance: a systematic review. Journal of gastrointestinal and liver diseases. JGLD. 2010; 19(4):409-414.

9. Lee JW, Kim N, Kim JM, Nam RH, Chang H, Kim JY, Shin CM, Park YS, Lee $\mathrm{DH}$, Jung HC. Prevalence of primary and secondary antimicrobial resistance of Helicobacter pylori in Korea from 2003 through 2012. Helicobacter. 2013;18(3):206-14.

10. Liu WZ, Xie Y, Lu H, Cheng H, Zeng ZR, Zhou LY, Chen Y, Wang JB, Du YQ, Lu NH. Fifth Chinese National Consensus Report on the management of Helicobacter pylori infection. Helicobacter. 2018;23(2):e12475.

11. Yu L, Luo L, Long X, Liang X, Ji Y, Chen Q, Song Y, Li X, Graham DY, Lu H. Susceptibility-guided therapy for Helicobacter pylori infection treatment failures. Therapeutic Adv Gastroenterol. 2019;12:1-10.

12. Pan J, Shi Z, Lin D, Yang N, Meng F, Lin L, Jin Z, Zhou Q, Wu J, Zhang J, et al. Is tailored therapy based on antibiotic susceptibility effective? a multicenter, open-label, randomized trial. Front Med. 2020;14:43-50.

13. Byambajav TO, Bira N, Choijamts G, Davaadorj D, Gantuya B, Sarantuya T, Sarantuya G, Enkhtsetseg A, Erdenetsogt D, Battulga A, et al. Initial trials with susceptibility-based and empiric anti-H. pylori therapies in Mongolia. Front Pharmacol. 2019;10:394.

14. Kwon YH, Jeon SW, Nam SY, Lee HS, Park JH. Efficacy of tailored therapy for Helicobacter pylori eradication based on clarithromycin resistance and survey of previous antibiotic exposure: A single-center prospective pilot study. Helicobacter. 2019;24(4):e12585.

15. Malfertheiner P, Megraud F, O'Morain CA, Gisbert JP, Kuipers EJ, Axon AT, Bazzoli F, Gasbarrini A, Atherton J, Graham DY, et al. Management of Helicobacter Pylori infection-the Maastricht V/Florence Consensus Report. Gut. 2017;66(1):6-30.

16. Perez Aldana L, Kato M, Nakagawa S, Kawarasaki M, Nagasako T, Mizushima T, Oda H, Kodaira J, Shimizu Y, Komatsu Y, et al. The relationship between consumption of antimicrobial agents and the prevalence of primary Helicobacter pylori resistance. Helicobacter. 2002;7(5):306-9.

17. Kuo CH, Hu HM, Kuo FC, Hsu PI, Chen A, Yu FJ, Tsai PY, Wu IC, Wang SW, Li CJ, et al. Efficacy of levofloxacin-based rescue therapy for Helicobacter pylori infection after standard triple therapy: a randomized controlled trial. Journal Antimicrob Chemother. 2009;63(5):1017-24.

18. Munoz N, Sanchez-Delgado J, Baylina M, Puig I, Lopez-Gongora S, Suarez $D$, Calvet X. Systematic review, meta-analysis, and meta-regression: successful second-line treatment for Helicobacter pylori. Helicobacter. 2018;23(3):e12488.

19. Osato MS, Reddy R, Reddy SG, Penland RL, Graham DY. Comparison of the Etest and the NCCLS-approved agar dilution method to detect metronidazole and clarithromycin resistant Helicobacter pylori. Int J Antimicrob Agents. 2001;17(1):39-44.

20. Cockerill FR. Clinical and laboratory standards institute performance standards for antimicrobial susceptibility testing: twenty-second informational supplement. National Committee Clinical Laboratory Standards. 2012;32:1-188.

21. Danielak D, Karazniewicz-Lada M, Wisniewska K, Bergus P, Burchardt $P$, Komosa A, Glowka F. Impact of CYP3A4*1G Allele on Clinical Pharmacokinetics and Pharmacodynamics of Clopidogrel. Eur J Drug Metab Pharmacokinet. 2017;42(1):99-107.

22. Jukic MM, Haslemo T, Molden E, Ingelman-Sundberg M. Impact of CYP2C19 Genotype on Escitalopram Exposure and Therapeutic Failure: A Retrospective Study Based on 2,087 Patients. Am J Psychiatr. 2018;175(5):463-70.

23. Zhang YX, Zhou LY, Song ZQ, Zhang JZ, He LH, Ding Y. Primary antibiotic resistance of Helicobacter pylori strains isolated from patients with dyspeptic symptoms in Beijing: a prospective serial study. World J Gastroenterol. 2015;21(9):2786-92.

24. Kim BJ, Kim HS, Song HJ, Chung IK, Kim GH, Kim BW, Shim KN, Jeon SW, Jung YJ, Yang CH, et al. Online Registry for Nationwide Database of Current Trend of Helicobacter pylori Eradication in Korea: Interim Analysis. J Korean Med Sci. 2016;31(8):1246-53.

25. Hori K, Miwa H, Matsumoto T. Efficacy of 2-week, second-line Helicobacter pylori eradication therapy using rabeprazole, amoxicillin, and metronidazole for the Japanese population. Helicobacter. 2011;16(3):234-40.

26. Georgopoulos SD, Ladas SD, Karatapanis S, Triantafyllou K, Spiliadi C, Mentis A, Artikis V, Raptis SA. Effectiveness of two quadruple, tetracycline- or 
clarithromycin-containing, second-line, Helicobacter pylori eradication therapies. Aliment Pharmacol Ther. 2002;16(3):569-75.

27. Chuah SK, Tai WC, Hsu PI, Wu DC, Wu KL, Kuo CM, Chiu YC, Hu ML, Chou YP, Kuo YH, et al. The efficacy of second-line anti-Helicobacter pylori therapy using an extended 14-day levofloxacin/amoxicillin/proton-pump inhibitor treatment-a pilot study. Helicobacter. 2012;17(5):374-81.

28. Jheng GH, Wu IC, Shih HY, Wu MC, Kuo FC, Hu HM, Liu CJ, Hsu WH, Hu CT, Bair MJ, et al. Comparison of Second-Line Quadruple Therapies with or without Bismuth for Helicobacter pylori Infection. Biomed Res Int. 2015;2015:163960.

29. Ueki N, Miyake K, Kusunoki M, Shindo T, Kawagoe T, Futagami S, Tsukui T, Inagaki H, Sakamoto C. Impact of quadruple regimen of clarithromycin added to metronidazole-containing triple therapy against Helicobacter pylori infection following clarithromycin-containing triple-therapy failure. Helicobacter. 2009;14(2):91-9.

30. Seyyedmajidi M, Abbasi L, Seyedmajidi S, Hosseini SA, Ahmadi A, Hajiebrahimi S, Vafaeimanesh J. Levofloxacin-containing triple therapy versus bismuth-based quadruple therapy as regimens for second line antiHelicobacter pylori. Caspian J Intern Med. 2019;10(2):211-6.

31. Gisbert JP, Romano M, Gravina AG, Solis-Munoz P, Bermejo F, MolinaInfante J, Castro-Fernandez M, Ortuno J, Lucendo AJ, Herranz M, et al. Helicobacter pylori second-line rescue therapy with levofloxacin- and bismuth-containing quadruple therapy, after failure of standard triple or non-bismuth quadruple treatments. Aliment Pharmacol Ther. 2015;41(8):768-75.

32. Liou JM, Chang CY, Chen MJ, Chen CC, Fang YJ, Lee JY, Wu JY, Luo JC, Liou TC, Chang WH, et al. The primary resistance of Helicobacter pylori in Taiwan after the national policy to restrict antibiotic consumption and its relation to virulence factors-a nationwide study. PLOS ONE. 2015;10(5):e0124199.

33. Hsu PI, Tsai FW, Kao SS, Hsu WH, Cheng JS, Peng NJ, Tsai KW, Hu HM, Wang YK, Chuah SK, et al. Ten-day quadruple therapy comprising proton pump inhibitor, bismuth, tetracycline, and levofloxacin is more effective than standard levofloxacin triple therapy in the second-line treatment of Helicobacter pylori Infection: a randomized controlled trial. Am J Gastroenterol. 2017;112(9):1374-81.

34. Qian J, Ye F, Zhang J, Yang YM, Tu HM, Jiang Q, Shang L, Pan XL, Shi RH, Zhang GX. Levofloxacin-containing triple and sequential therapy or standard sequential therapy as the first line treatment for Helicobacter pylori eradication in China. Helicobacter. 2012;17(6):478-85.

35. Miftahussurur M, Aftab H, Shrestha PK, Sharma RP, Subsomwong $P_{,}$ Waskito LA, Doohan D, Fauzia KA, Yamaoka Y. Effective therapeutic regimens in two South Asian countries with high resistance to major Helicobacter pylori antibiotics. Antimicrob Resistance Infect Cont. 2019:8:40.

36. Sotoudehmanesh R, Malekzadeh R, Vahedi H, Dariani NE, Asgari AA, Massarrat S. Second-line Helicobacter pylori eradication with a furazolidonebased regimen in patients who have failed a metronidazole-based regimen. Digestion. 2001;64(4):222-5.

37. Kuo CH, Lu CY, Shih HY, Liu CJ, Wu MC, Hu HM, Hsu WH, Yu FJ, Wu DC, Kuo FC. CYP2C19 polymorphism influences Helicobacter pylori eradication. World J Gastroenterol. 2014;20(43):16029-36.

\section{Publisher's Note}

Springer Nature remains neutral with regard to jurisdictional claims in published maps and institutional affiliations.
Ready to submit your research? Choose BMC and benefit from:

- fast, convenient online submission

- thorough peer review by experienced researchers in your field

- rapid publication on acceptance

- support for research data, including large and complex data types

- gold Open Access which fosters wider collaboration and increased citations

- maximum visibility for your research: over $100 \mathrm{M}$ website views per year

At BMC, research is always in progress.

Learn more biomedcentral.com/submissions 